\title{
Paramedian thalamic syndrome due to occlusion of the artery of Percheron: A rare case of stroke
}

\author{
W S Weerasinghe ${ }^{1}$, S A M Kularatne ${ }^{2}$, L P M M K Pathirage ${ }^{2}$ \\ (Index words: Brain stem stroke, Paramedian thalamic syndrome, Artery of Percheron)
}

Ceylon Medical Journal 2018; 63: 30-31

DOI: http://doi.org//10.4038/cmj.v64i1.8835

\section{Case presentation and discussion}

A 35-year-old previously healthy male found unresponsive by his friends despite many attempts to wake him up from the sleep in the morning and brought to the Teaching Hospital, Peradeniya on May 2018. He went to sleep on a thin mattress spread on the ground at about $11 \mathrm{pm}$ at a mosque. His friends denied the use of alcohol or illicit drugs at the night and could not find a snake or bite mark in the body, or nor any remnants of drugs or other substance that would have been abused and they were not involved in indoor cooking.

On admission his vitals were normal, but he was drowsy with Glasgow Coma Scale (GCS) score of 9/15 (E- 2, V-2, M-5), the pupils were unequal, right side $3 \mathrm{~mm}$ and left $1 \mathrm{~mm}$ in size with sluggish light reaction. There was bilateral ptosis more on the right side with oculomotor nerve palsy. His motor power was $>4+$ in both upper and lower limbs and planters were up-going on the right side. An urgent non-contrast CT brain was performed followed by contrast-enhanced CT (CECT) of the brain, which showed a hypodense area in right frontal lobe suggestive of an old infarction. Due to low GCS, he was electively intubated and transferred to the intensive care unit (ICU). His urine toxicology screen, EEG, CSF studies and tests for myasthenia gravis was normal. MRI done five days later showed features of a sub-acute infarction in paramedian thalami and rostral midbrain bilaterally (right >left) (Figure 1) compatible with the paramedian thalamic syndrome (PTS). He was started on aspirin and a statin, and made a gradual recovery until he was lost to followup.

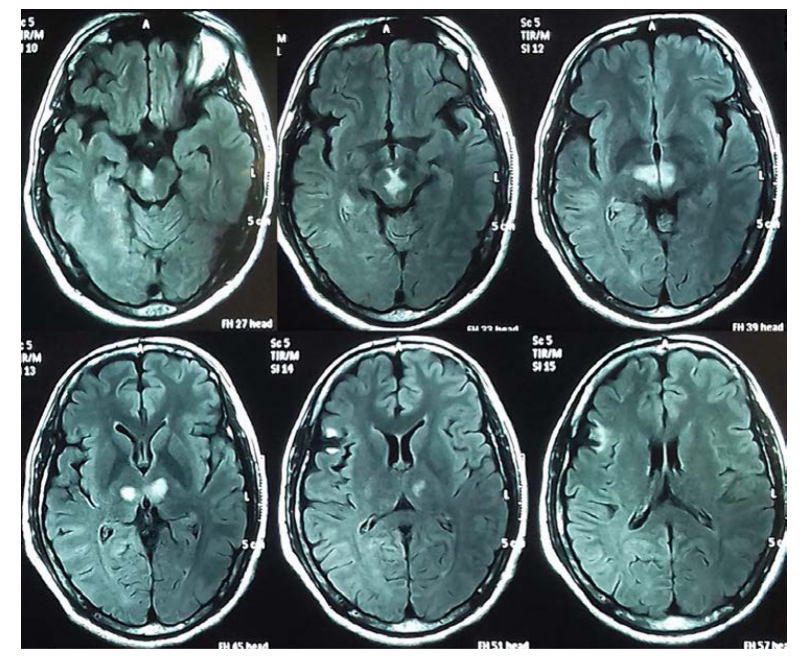

Figure 1. MRI brain T2, FLAIR hyper intensity with no contrast enhancement in the paramedian thalami and rostral mid brain bilaterally (Right >left), T2, FLAIR hyper intensity with no diffusion restriction and contrast enhancement is also seen in right frontal lobe.

A French medical scientist Gerard Percheron, 1973 [1] first described an anatomic variant of the paramedian artery which arises from the P1 section of the posterior cerebral artery and supplies bilateral thalamus from a single trunk called artery of Percheron (AOP) [2] (Figure 2). Occlusion of this artery can give rise to bilateral paramedian thalamic infarcts called PTS. The classic triad of hypersomnolence, ocular disturbances (supranuclear and

${ }^{1}$ Teaching Hospital, Peradeniya, ${ }^{2}$ Faculty of Medicine, University of Peradeniya, Peradeniya, Sri Lanka.

Correspondence: WSW, e-mail:<sunethws@hotmail.com>. Received 20 December 2018 and revised version accepted 06 March 2019.

(i) This is an open-access article distributed under the terms of the Creative Commons Attribution License, which permits unrestricted use, distribution, and reproduction in any medium, provided the original author and source are credited. 
vertical gaze palsy) and neuropsychological deficits (including an amnestic syndrome) have been described in these patients [3]. We conclude that a great degree of awareness of stroke syndromes can improve patient outcome with early recognition and suitable treatment option like thrombolysis or vascular interventions.
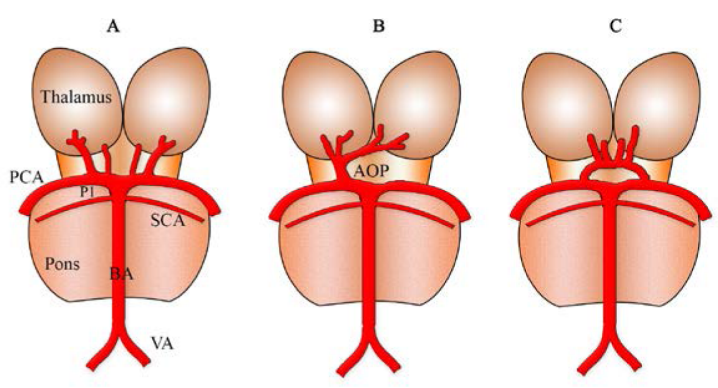

Figure 2. Known variations of the paramedian thalamic-mesencephalic arterial supply.

A. The commonest variation, many small perforating arteries arising from P1 segment of the PCA.

B. The artery of Percheron, a single perforating blood vessel arising from one P1 segment.

C. The other variation, a bridging artery from both P1 segments provide arcade of perforating branches.

PCA - Posterior Cerebral Artery; BA - Basilar Artery;

SCA - Superior Cerebellar Artery; AOP - Artery of Percheron;

VA - Vertebral Artery.

\section{Declarations}

Ethics approval and consent to participate

Written informed consent was obtained from the patient for publication of this case report and any accompanying images. A copy of the written consent is available for review with the Editor-in-Chief of this Journal.

\section{Consent for publication}

Written informed consent was obtained from the patient for publication of this case report.

\section{Authors' contributions}

All made equal contribution to the identification of the patient, conception of the work, diagnosis and treatment, drafting the manuscript, writing references, revising it critically for important intellectual content, final development of case report. All authors read and approved the final manuscript.

\section{Acknowledgements}

We acknowledge the patient, Dr Ganganath Rodrigo (Consultant Radiologist - THK) and his team in the Department of Radiology at the Teaching Hospital Kandy.

\section{References}

1. Percheron G. The anatomy of the arterial supply of the human thalamus and its use for the interpretation of the thalamic vascular pathology. Z Neurol 1973; 205(1): 1-13.

2. Matheus MG, Castillo M. Imaging of acute bilateral paramedian thalamic and mesencephalic infarcts. AJNR Am J Neuroradiol 2003; 4(10): 2005-8.

3. Kostanian V, Cramer SC, Castillo M, Fischbein NJ, Glastonbury CM, Hildenbrand PG, et al. Artery of Percheron thrombolysis. AJNR Am J Neuroradiol 2007; 28(5): 870-1. 